\title{
Papel de las tiazidas en la profilaxis de la litiasis cálcica recidivante
}

\author{
A. Fernández-Rodríguez, M. Arrabal-Martín*, Ma J. García-Ruiz, M. A. Arrabal-Polo*, \\ S. Pichardo-Pichardo, A. Zuluaga-Gómez*.
}

Hospital San Juan de la Cruz. Úbeda (Jaén). Departamento de Cirugía, *Universidad de Granada.

Actas Urol Esp 2006; 30 (3): 305-309

\section{RESUME N}

PAPEL DE LAS TIAZIDAS EN LA PROFILAXIS DE LA LITIASIS CÁLCICA RECIDIVANTE

Objetivo: Demostrar la eficacia prolongada de las tiazidas en la profilaxis y tratamiento de las recidivas en pacientes con litiasis cálcica de oxalato y fosfato cálcico.

Métodos: Se realiza un estudio prospectivo aleatorizado, con un seguimiento de tres años, en 150 pacientes diagnosticados de litiasis cálcica recidivante. Los pacientes se distribuyen en tres grupos: A) 50 casos sometidos a observación sin tratamiento, B) 50 casos tratados con $50 \mathrm{mg} /$ día de hidroclorotiazida y C) 50 casos tratados con $50 \mathrm{mg}$ de hidroclorotiazida y $20 \mathrm{mlEq}$ de citrato potásico/día. En cada grupo se realiza estudio renal con técnicas de imagen, y estudio metabólico urinario basal, 12, 24 y 36 meses.

Resultados: En los pacientes tratados con tiazidas (Grupo B y C) se obtiene una reducción significativa de recidiva litiásica en relación con el grupo control (Grupo A). La alteración más frecuente encontrada en el estudio metabólico fue hipercalciuria, 52\% de los casos; el 16\% presentan patrón litógeno mixto. El número de recidivas y necesidad de nuevas sesiones de litotricia extracorpórea en los pacientes con hipercalciuria tratados con tiazidas disminuye significativamente con respecto al Grupo A $(\mathrm{p}=0.003)$

Conclusiones: Se observa una relación significativa entre patrón litógeno y recidiva litiásica. Las tiazidas nos ayudan a controlar los factores litogénicos y las recidivas en pacientes con litiasis cálcica. Este efecto es prolongado y significativo en pacientes con hipercalciuria.

Palabras clave: Prevención. Recidiva. Litiasis renal.

\section{ABSTRACT}

THE ROLE OF THIAZIDES IN THE PROPHYLAXIS OF RECURRENT CALCIUM LITHIASIS

Objective: To show the prolonged efficacy of thiazides in the prophylaxis and treatment of recurrences in patients with calcium oxalate and phosphate lithiasis.

Methods: A randomised prospective study is conducted, with a three-year follow-up, in 150 patients diagnosed with recurrent calcium lithiasis. The patients are divided into three groups: A) 50 cases subject to observation with no treatment, B) 50 cases treated with $50 \mathrm{mg} /$ day of hydrochlorothiazide, and C) 50 cases treated with $50 \mathrm{mg}$ of hydrochlorothiazide and $20 \mathrm{mlEq}$ of potassium citrate/day. Each group is subject to a renal study with imaging techniques and a urinary metabolic study at baseline, 12,24 and 36 months.

Results: The patients treated with thiazides (Groups B and C) obtain a significant reduction in lithiasis recurrence compared with the control group (Group A). The most common abnormality found in the metabolic study was hypercalciuria, $52 \%$ of cases; $16 \%$ present a mixed lithogenic pattern. The number of recurrences and need for new sessions of extracorporeal lithotripsy in patients with hypercalciuria treated with thiazides is significantly smaller than in Group A $(p=0.003)$

Conclusions: We observe a significant relation between lithogenic pattern and lithiasis recurrence. Thiazides help us to control lithogenic factors and recurrences in patients with calcium lithiasis. This effect is prolonged and significant in patients with hypercalciuria.

Key words: Prevention. Recidiva. Renal lithiasis.

Abreviaturas: LEOC.- Litotricia extracorpórea por ondas de choque. EMU.- Estudio Metabólico Urinario 
$\mathrm{L}$ a litiasis urinaria, por su elevada incidencia y prevalencia, constituye un problema sanitario a pesar de la litotricia endoscópica y extracorpórea $^{1}$, debemos analizar los factores de riesgo litogénico y aplicar mecanismos correctores ${ }^{2}$, que controlen el elevado porcentaje de recidivas, hasta el $80 \%$ de los casos en el transcurso de la vida $^{3}$. En estos pacientes se pueden detectar factores litogénicos que deben ser corregidos ${ }^{4}$.

Los diuréticos tiazídicos han sido utilizados para el tratamiento médico de la litiasis cálcica hipercalciúrica, en base a su capacidad para reducir la excreción renal de calcio. Algunos autores consideran que este efecto no es permanente y le atribuyen una serie de efectos adversos, hipotensión, hipocitraturia, e hiperuricemia $^{5}$. Nuestro objetivo es demostrar la eficacia prolongada de las tiazidas en la profilaxis y tratamiento de las recidivas en pacientes con litiasis cálcica de oxalato y fosfato cálcico.

\section{MATERIAL Y MÉTODOS}

Se realiza un estudio prospectivo, durante 36 meses en 150 pacientes diagnosticados de litiasis cálcica recidivante, distribuidos en tres grupos:

Grupo A: Observamos evolución sin tratamiento médico.

Grupo B: Tratados con tiazidas (hidroclortiazida, $50 \mathrm{mg} /$ día).

Grupo C: Tratados con tiazidas y citrato potásico, $20 \mathrm{mlEq} /$ día.

Los pacientes se asignaron de forma aleatoria a cada grupo. Los criterios de inclusión establecidos para este estudio son:

1. Pacientes de ambos sexos, edades entre 1865 años.

2. Haber presentado más de un episodio de litiasis cálcica en los últimos 36 meses, resuelto tras: expulsión del cálculo, Litotricia Extracorpórea por Ondas de Choque (LEOC), Cirugía. (Endoscópica y/o Abierta).

3. Función y morfología renal normal. Ausencia enfermedad endocrina.

En todos los pacientes se realiza examen basal: Estudio metabólico urinario (EMU), radiografía simple aparato urinario, urograma y/o ecografía renal. Análisis del cálculo. Durante los tres años de seguimiento: EMU a los 12-24-36 meses, Rx. simple a los 6-12-18-24-30-36 meses.
- Método de Estudio Metabólico Urinario. Para el EMU se utiliza un programa informatizado que recoge datos séricos (creatinina, calcio, fósforo, ácido úrico.....) y urinarios (creatinina, calcio, fósforo, ácido úrico, magnesio, citratos....), a partir de ellos se obtienen indicadores e índices que permiten obtener las siguientes ayudas de diagnóstico:

Hipercalciuria: Absortiva-excretora-resortiva. Hiperoxaluria: Absortiva-endógena.

Hiperuricosuria: Endógena-enterorrenal.

Hipocitraturia. Hipomagnesuria. Acidosis tubular renal.

Se considera patrón litógeno, a la detección en el EMU de una o varias alteraciones: Hipercalciuria absortiva o renal, Hipocitraturia, Hiperoxaluria, Hiperuricosuria. Si existe más de una alteración se define como patrón litógeno mixto.

- Análisis del cálculo. Estudio con microscopio petrográfico y espectrografía infrarroja.

- Metodología estadística. En el estudio estadístico inferencial se aplica el test de la Chi cuadrado y test de Fisher, se acepta significación estadística si $\mathrm{p}<0.05$.

\section{RESULTADOS}

Presentamos los resultados obtenidos en cada uno de los grupos (A, B y C) desde un punto de vista descriptivo e inferencial.

\section{GRUPO A: GRUPO CONTROL}

En este grupo recidivan 28 pacientes, 56\%. Generalmente las recidivas se localizan en cáliz medio e inferior (86\%). La evolución del patrón litógeno se expone en la Tabla 1. Podemos apreciar como durante el periodo de seguimiento se mantienen los porcentajes de las distintas alteraciones metabólicas.

Durante el periodo de seguimiento se produce expulsión espontánea de las recidivas en 2 casos. Se ha producido evolución satisfactoria en el $48 \%$ de los pacientes y se realiza litotricia extracorpórea en 13 casos.

\section{GRUPO B: ENFERMOS TRATADOS CON TIAZIDAS}

En este grupo recidivan 16 pacientes, 32\%. El $100 \%$ de las recidivas se localizan en cáliz medio e inferior. La evolución del patrón metabólico se expone en la Tabla 2. Podemos apreciar como 
Tabla 1

Patrón metabólico (EMU). Basal, 12, 24, 36 meses. Grupo A.

\begin{tabular}{lcccc}
\hline Patrón litógeno & Basal & $\mathbf{1 2}$ meses & $\mathbf{2 4}$ meses & $\mathbf{3 6}$ meses \\
\hline Hipercalciuria absortiva o renal & $34 \%$ & $26 \%$ & $38 \%$ & $30 \%$ \\
Hipocitraturia & $14 \%$ & $14 \%$ & $10 \%$ & $14 \%$ \\
Hiperuricuria & $4 \%$ & $2 \%$ & $6 \%$ & $4 \%$ \\
Hiperoxaluria & $2 \%$ & $4 \%$ & $4 \%$ & $4 \%$ \\
Mixto & $14 \%$ & $16 \%$ & $14 \%$ & $16 \%$ \\
Sin trastorno & $32 \%$ & $38 \%$ & $28 \%$ & $32 \%$ \\
\hline
\end{tabular}

Tabla 2

Patrón metabólico basal, 12, 24, 36 meses. Grupo B.

\begin{tabular}{lcccc}
\hline Patrón litógeno & Basal & 12 meses & 24 meses & $\mathbf{3 6}$ meses \\
\hline Hipercalciuria absortiva o renal & $42 \%$ & $16 \%$ & $10 \%$ & $16 \%$ \\
Hipocitraturia & $14 \%$ & $22 \%$ & $24 \%$ & $28 \%$ \\
Hiperuricuria & $2 \%$ & & $2 \%$ & $4 \%$ \\
Mixto & $12 \%$ & $6 \%$ & - & - \\
Sin trastorno & $30 \%$ & $56 \%$ & $64 \%$ & $52 \%$ \\
\hline
\end{tabular}

durante el periodo de seguimiento desciende el porcentaje de hipercalciuria, se incrementa el número de enfermos con hipocitraturia y al final del estudio el número de enfermos sin alteración metabólica pasa del 30 al $52 \%$ y no se detecta patrón litógeno mixto.

Durante el periodo de seguimiento se produce expulsión espontánea de las recidivas en 8 casos. Se ha obtenido éxito terapéutico en el $84 \%$ de los pacientes y se realiza litotricia extracorpórea en 4 casos.

\section{GRUPO C: ENFERMOS TRATADOS CON TIACIDAS Y CITRATO POTÁSICO}

En este grupo recidivan 15 pacientes, 30\%. Generalmente las recidivas se localizan en cáliz medio e inferior (90\%). La evolución del patrón metabólico se expone en la Tabla 3. Podemos apreciar como durante el periodo de seguimiento desciende el porcentaje de hipercalciuria, patrón litógeno mixto e hipocitraturia y al finalizar el estudio el número de pacientes sin alteración metabólica pasa del 26 al 66\%.

Durante el periodo de seguimiento se produce expulsión espontánea de las recidivas en 6 casos. Se ha obtenido éxito terapéutico en el $82 \%$ de los pacientes y se realiza litotricia extracorpórea en 4 casos.

\section{ANÁLISIS ESTADÍSTICO} INFERENCIAL

Existe una relación estadísticamente significativa entre la presencia de patrón litógeno y la recidiva litiásica. $\left(\chi^{2}\right.$ exp. $\left.=5,939, p=0,018\right)$. En los pacientes tratados con tiazidas la recidiva litiásica se produce en el 30$32 \%$ de los casos frente al $56 \%$ del grupo control, diferencia estadísticamente significativa $\left(\chi^{2}\right.$ exp. $\left.p=0,016\right)$, no hay diferencias entre los grupos B-C. Entre los grupos B-C solo se observan diferencias significativas en la evolución de la hipocitraturia, se duplica en el grupo B y desciende a la mitad en el grupo $\mathrm{C}$.

Durante el periodo de seguimiento se realiza litotricia extracorpórea en el $26 \%$ de los pacientes del grupo A, 8\% del grupo B y 8\% del grupo C. Diferencias estadísticamente significativas. $\left(\chi^{2}\right.$ exp. $\left.p=0,018\right)$.

Resultados en pacientes con hipercalciuria: Presentan hipercalciuria, 17 pacientes del grupo A, 21 del grupo B y 14 del grupo C. En los grupos B-C tratados con tiazidas presentan recidivas el 19\% (4 casos) y el 7\% (1 caso) respectivamente frente al 59\% (10 casos) del grupo A, diferencias con elevada significación estadística $(p=0,003$, test de Fisher) y la necesidad de litotricia extracorpórea durante el periodo de seguimiento, en estos pacientes (LEOC en 3 casos del grupo B y 0
Tabla 3

Patrón metabólico basal, 12, 24, 36 meses. Grupo C.

\begin{tabular}{lcccc}
\hline Patrón litógeno & Basal & $\mathbf{1 2}$ meses & 24 meses & $\mathbf{3 6}$ meses \\
\hline Hipercalciuria absortiva o renal & $28 \%$ & $4 \%$ & $4 \%$ & $6 \%$ \\
Hipocitraturia & $16 \%$ & $16 \%$ & $10 \%$ & $8 \%$ \\
Hiperuricuria & $6 \%$ & $16 \%$ & $10 \%$ & $8 \%$ \\
Hiperoxaluria & $2 \%$ & $2 \%$ & - & - \\
Mixto & $22 \%$ & $4 \%$ & $8 \%$ & $12 \%$ \\
Sin trastorno & $26 \%$ & $58 \%$ & $68 \%$ & $66 \%$ \\
\hline
\end{tabular}


casos del grupo C) fue significativamente inferior en relación con el subgrupo de pacientes hipercalciúricos del grupo A (LEOC en 6 casos) ( $p=0.032$, test de Fisher), los mejores resultados se obtienen en el grupo C.

\section{COMENTARIOS}

La acción fundamental que las tiazidas ejercen en beneficio de la profilaxis de litiasis cálcica es la inducción de hipocalciuria ${ }^{6}$, algunos autores como Yendt ${ }^{7}$, han intentado demostrar sin resultados concluyentes, un efecto directo de las tiazidas sobre la absorción de calcio intestinal en pacientes con hipercalciuria absortiva.

En nuestro estudio sobre 150 pacientes con litiasis cálcica, las recidivas y necesidad de nuevas sesiones de LEOC han sido menores en los pacientes tratados con tiazidas (grupos $\mathrm{B}-\mathrm{C}$ ) frente a los controles (grupo A), con diferencias significativas.

En el análisis descriptivo, observamos que en el grupo A se produce recidiva litiásica en el 56\% de los casos. El patrón litógeno es similar desde el inicio hasta el final del estudio. Se realiza LEOC en 13 casos (26\%).

En el grupo B se produce recidiva en el 32\% de los casos. Disminuye el riesgo litógeno, 52\% de pacientes sin alteración metabólica al final del estudio. Se realiza LEOC en 4 casos (8\%).

En el grupo C se produce recidiva en el 30\% de los casos. Disminuye de forma sensible el riesgo litógeno, $66 \%$ de pacientes sin alteración metabólica al final del estudio. Se realiza LEOC en 4 casos (8\%).

En este estudio se ha demostrado que existe una relación estadísticamente significativa entre patrón litógeno $\mathrm{y}$ recidiva litiásica, $\mathrm{p}=0,018$. Coincidimos con $\mathrm{Pak}^{8}$ en que la mayor parte de los pacientes con cálculos renales de oxalato cálcico presentan al menos una alteración metabólica. El trastorno metabólico que se asocia con más frecuencia a litiasis cálcica es hipercalciuria, según algunos estudios puede situarse esta alteración entre el 30-60\% ${ }^{9}$. En nuestro estudio presentan hipercalciuria aislada o incluida en el patrón litógeno mixto el $52 \%$ de pacientes, (se detecta patrón litógeno mixto en el 16\% de estudios basales).

El número de recidivas en pacientes con hipercalciuria tratados con tiazidas disminuye significativamente con respecto al grupo A $(\mathrm{p}=0,003)$, en estos pacientes comprobamos que la necesidad de LEOC en el grupo A fue superior al doble que en los pacientes hipercalciúricos de los grupos sometidos a tratamiento con tiazidas $(\mathrm{p}=0,032)$.

Los pacientes que presentan alteración metabólica urinaria en el estudio basal, tratados con tiazidas con o sin citrato potásico presentan una respuesta terapéutica superior a la del grupo A $(p=0,001)$, siendo aún mayor en los pacientes con hipercalciuria y patrón litógeno mixto, $\mathrm{Coe}^{10}$ observa en 78 pacientes con litiasis cálcica tratados con tiazidas una reducción del porcentaje de recidivas, y Pack ${ }^{11}$ encuentra una reducción muy elevada (93\%) de recidivas en 27 pacientes tratados durante un periodo de tres años. En los casos que presentan patrón litógeno mixto puede recomendarse la asociación de otros fármacos como el alopurinol, concretamente en hipercalciurias asociadas a hiperuricosurias, $\mathrm{o}$ incluso en hiperuricosurias asociadas a hiperoxalurias ${ }^{12}$.

Además del efecto hipocalciúrico de las tiazidas, estos fármacos presentan otros efectos que pueden tener trascendencia en el proceso litogénico, así algunos autores ${ }^{13}$ encuentran una disminución de los niveles de oxalato urinario en tratamientos prolongados durante 12 meses. Por otro lado las tiazidas parecen estar implicadas en el aumento de la excreción de cinc y magnesio por orina, reconocidos como inhibidores de la cristalización ${ }^{7,14}$.

El efecto hipocalciúrico de las tiazidas en tratamientos prolongados (18-24 meses) puede tener una acción limitada con posible retorno de la calciuria en algunos casos a niveles previos al tratamiento, sobre todo en las hipercalciurias absortivas ${ }^{15}$, esto parece debido a que al aumentar la calcemia se estimularían las células $\mathrm{C}$ del tiroides con producción de calcitonina que aumentaría la excreción urinaria de calcio ${ }^{16}$. Nosotros hemos observado este efecto limitante de las tiazidas en el $26 \%$ y $23 \%$ de los pacientes en los grupos B y C respectivamente, con recuperación posterior de la acción hipocalciúrica a partir de los 24 meses y sin necesidad de "vacaciones terapéuticas".

En el momento actual se considera a las tiazidas como el tratamiento de primera línea en 
hipercalciurias absortivas ${ }^{17}$, también en el resto de hipercalciurias, sólo existe una excepción y es la hipercalciuria del hiperparatiroidismo primario. Además y este es un dato importante a nuestro juicio, se ha demostrado que las tiazidas reducen la incidencia de cálculos en pacientes no seleccionados con litiasis cálcica ${ }^{18}$.

La hipocitraturia sola o combinada con otros trastornos metabólicos se observó en el $20 \%$ de los estudios básales realizados en los pacientes estudiados. Es cierto que se ha demostrado una mejoría en cuanto a la tasa de formación de cálculos con el tratamiento exclusivo de citrato potásico ${ }^{17,19}$, sin embargo coincidimos con otros autore ${ }^{20}$ en que su efecto es mayor en los casos de hipocitraturia, en este estudio se demuestra su utilidad en la corrección de la hipocitraturia inducida por tiazidas.

\section{CONCLUSIONES}

Se observa una relación significativa entre patrón litógeno y recidiva litiásica. Las tiazidas nos ayudan a controlar los factores litogénicos y las recidivas en pacientes con litiasis cálcica. Este efecto es prolongado y significativo en pacientes con hipercalciuria.

\section{REFERENCIAS}

1. Arrabal Martín M. Grupo de litiasis AEU.: Extracorporeal lithotripsy in Spain in the 20 th Century. Actas Urol Esp 2000;24:699-708.

2. Pak CYC. Citrate and renal calculi. Miner Electrolyte Metab. 1978;13:257-266.

3. Norlin A, Lindell B, Granberg PO, Lindvall N. Urolithiasis. A study of its frequency. Scand J Urol Nephrol 1976;10:150153.

4. Pak CYC. Role of medical prevention. J Urol. 1989;141:798801.

5. Costanzo LS, Weiner IM. On the hypocalciuric action of chlorothiazide. J Clin Invest 1974;54:628-637.

6. Ettinger B, Citron JT, Livermore B, Dolman LI. Chlorthalidone reduces calcium oxalate calculous recurrence but magnesium hydroxide does not. J Urol 1988;139: 679684 .
7. Yendt ER, Cohaim M. Prevention of calcium stones with thiazides. Kidney Int 1978;13:397-409.

8. Pak CYC. Sodium cellulose phosphate: mechanism of action an effect of mineral metabolism. J Clin Pharmacol New Drugs 2001;13:15-27.

9. Ruml LA, Pearle MS, Pak, CYC. Medical therapy, calcium oxalate urolithiasis. Urol Clin North Am 1997;24:117-133.

10. Coe FL.: Treated and untreated recurrent calcium nephrolithiasis in patients with idiopathic hypercalciuria, hyperuricosuria, or no metabolic disorder. Ann Intern Med. 1977; 87:404-410.

11. PAK CYC, Peters P, Hurt G, Kadesky M, Fine M, Reisman D. et al. Is selective therapy of recurrent nephrolithiasis possible? Am J Med 1981;71:615-622.

12. Okada Y, Nonomura M, Takeuchi H, Kawamura J, Yoshida O. Experimental and clinical studies on calcium lithiasis. Prevention of recurrent calcium stones with thiazides and allopurinol. Hinyo K. 1986;32:1247-1257.

13. Elomaa I, Ala-Opas M, Porkka L. Five years of experience with selective therapy in recurrent calcium nephrolithiasis. J Urol 1984;132:656-661.

14. Laerum E. Metabolic effects of thiazide versus placebo in patients under long-term treatment for recurrent urolithiasis. Scand J Urol Nephrol 1984;18:143-149.

15. Preminger GM, Pak CYC. Eventual attenuation of hypercalciuric response to hydrochlorothiazide in absorptive hypercalciuria. J Urol 1987;137:1104-1109.

16. Arrabal M, Lancina A. Tratamiento médico de la enfermedad litiásica. in Arrabal, M.; Lancina, A.; García, M.: Criterios clínicos y tratamiento actual de la litiasis urinaria. ENE Ediciones SA, Madrid. 1990; pag. 91-111.

17. Pak CYC. Citrate and renal calculi: new insights and future directions. Am Kidney Dis 1991;17:420-425.

18. Ahlstrand C, Tiselius HG, Larson L, Hellgren E. Clinical experience with long term bendroflumethiazide in patients with recurrentrenal calcium oxalate stone formers. $\mathrm{Br} \mathrm{J}$ Urol 1984;56:255-262.

19. Rudman D, Kutner MH, Redd SC $2^{\text {nd }}$, Waters WC 4 th., Gerron GG, Bleier J. Hypocitraturia in calcium nephrolithiasis. J Clin End Metab 1982;55:1052-1057.

20. Abdulhadi MH, Hall PM, Streem SB. Can citrate therapy prevent nephrolithiasis?. Urology 1993;41:221-224.

Dr. Miguel Arrabal Martín

E-mail: arrabalm@supercable.es

(Trabajo recibido el 23 de noviembre de 2005) 